\title{
Emergency Vehicle Scheduling Problem with Time Utility in Disasters
}

\author{
Xiaobing Gan, ${ }^{1}$ Yan Wang, ${ }^{1}$ Junbiao Kuang, ${ }^{1}$ Ye Yu, ${ }^{1}$ and Ben Niu ${ }^{1,2}$ \\ ${ }^{1}$ College of Management, Shenzhen University, Shenzhen 518060, China \\ ${ }^{2}$ Hefei Institute of Intelligent Machine, Chinese Academy of Sciences, Hefei 230031, China \\ Correspondence should be addressed to Xiaobing Gan; ganxb2001@163.com and Ben Niu; drniuben@gmail.com
}

Received 14 August 2014; Accepted 8 October 2014

Academic Editor: Yuping Wang

Copyright (c) 2015 Xiaobing Gan et al. This is an open access article distributed under the Creative Commons Attribution License, which permits unrestricted use, distribution, and reproduction in any medium, provided the original work is properly cited.

\begin{abstract}
This paper presents a flexible emergency rescue system which is chiefly composed of three parts, namely, disaster assistance center, relief vehicles, and disaster areas. A novel objective of utility maximization is used to evaluate the entire system in disasters. Considering the uncertain road conditions in the relief distribution, we implement triangular fuzzy number to calculate the vehicle velocity. As a consequence, a fuzzy mathematical model is built to maximize the utility of emergency rescue system and then converted to the crisp counterpart. Finally, the results of numerical experiments obtained by particle swarm optimization (PSO) prove the validity of this proposed mathematical model.
\end{abstract}

\section{Introduction}

The vehicle routing problem (VRP) can usually be described as choosing a better route travelled by the given vehicle so as to meet the customer demands efficiently. And the emergency vehicle scheduling problem (EVSP) is a special kind of vehicle routing problem as a result of the urgency of its service time. With natural disasters occurring frequently in recent years, EVSP has aroused soaring concerns. Özdamar et al. [1] viewed vehicles as commodities and built a model to minimize the delay. The improved Lagrangian relaxation is used to solve the subproblem. Tian et al. [2] considered the fuzzy demands and applied particle swarm optimization (PSO) to tackle the multiobjective problem. Moreover, Sheu [3] introduced a three-layer supply network and applied a hybrid fuzzy grouping method to cope with the distribution operation from perspectives of minimizing the costs and maximizing the demand fill rate synchronously.

As we all know, the importance of costs incurred in emergency rescue is weakened and the minimization of arrival time becomes an indicator of whether the emergency rescue system is effective and efficient. Sun et al. [4] proposed an emergency location-routing problem and aimed at minimizing the total time and cost. Ji and Zhu [5] introduced the salvable degree to emergency supply chain and a multiobjective optimization model is developed to minimize travel time and satisfy the demands of affected areas as much as possible. However, it is not enough to use the indicator of time or distance to evaluate an emergency rescue system. Therefore, based on the previous work [6], we take time utility into account and develop an improved model. Besides, two novel conceptions of efficiency risk and security risk are involved to weigh the road conditions in the distribution.

There are many methods used to solve EVSP problem. Yamada [7] modeled a city evacuation planning and then applied network flow theory to optimize this problem. Compared with traditional optimization approach, evolutionary computation has been studied widely in recent years. For instance, Tuson et al. [8] presented an evolutionary and metaheuristic optimization technique for emergency resource redistribution in the developing world. Yi and Kumar [9] introduced a metaheuristic of ant colony optimization to solve the emergency logistics problem and obtained a series of excellent solutions. Similarly, PSO algorithm performs better when employed to solve some NP-hard problems $[2,6]$. So we adopt PSO to tackle a case of EVSP in this paper on account of its excellent property. 


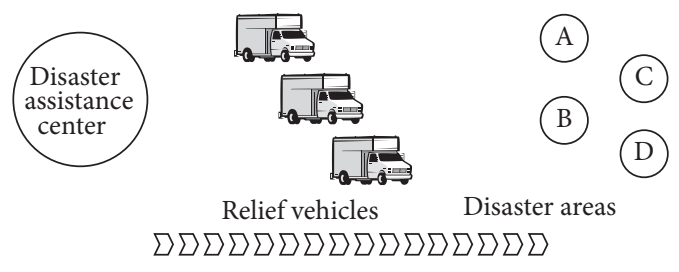

Figure 1: The emergency rescue system.

The main contributions of this paper are threefold.

(1) We introduce utility maximization to evaluate an emergency rescue system.

(2) Considering the uncertain road conditions, a fuzzy EVSP is modeled.

(3) By conducting contrast experiments, we validate the influence of efficient risk and security risk on the emergency rescue system.

In the remainder of this paper, Section 2 gives a brief introduction to EVSP. Section 3 aims to formulate a fuzzy model and convert it to a crisp model by using triangular fuzzy number. Section 4 presents PSO algorithm and constraint operation in the fitness function. Thereafter, the numerical experiments are conducted in Section 5. And conclusions are given in Section 6.

\section{Description of EVSP}

2.1. The Emergency Rescue System. The emergency rescue system is presented visually in Figure 1, including disaster assistance center, relief vehicles (containing relief commodities and relief workers), and disaster areas. First of all, the disaster assistance center serves as a command in terms of dispatching relief vehicles reasonably. The negative utility for the center is incurred by transportation costs. Secondly, the relief vehicles and workers suffer from the unexpected accident in the distribution, for example, aftershock and landslide. So we take security risk into account and it results in negative utility for relief vehicles. Finally, disaster areas obtain positive utility when receiving relief commodities. There is a trade-off in these three parts and the objective is to maximize the system utility.

We assume that the disaster assistance center with $K$ vehicles needs to distribute relief commodities to $n$ disaster area. Let $q_{k}$ denote the given capacity of vehicle $k$. The urgency degree of material demands for the $i$ th disaster area is $\beta_{i}\left(\beta_{i} \in(0,1), \sum_{i=1}^{n} \beta_{i}=1\right)$ and the corresponding demands are $r_{i}$. And the deadline of the $i$ th disaster area to receive the relief is $l t_{i}$. Besides, the unit freight is described as $c_{k}$ and the distance between $i$ and $j$ is $d_{i j}$. The actual utility received and unloading time of disaster area $i$ are denoted as $u_{i}\left(u_{0}\right.$ means the initial utility of each disaster area) and $s_{i}$, respectively. The parameter $b$ indicates the conversion factor which transforms transportation costs into the corresponding utility. So EVSP can be described as tracing a preferable route travelled by the rescue vehicles with limited capacity $\left(q_{k}\right)$. The assumptions are as follows [6].
(1) Firstly, there is only one disaster assistance center.

(2) Secondly, each disaster area must be served by exactly one vehicle. Correspondingly, each route between disaster areas must be traversed by only one relief vehicle.

(3) Thirdly, the demands of each disaster area must not exceed the assigned vehicle capacity.

(4) Finally, each vehicle must arrive at the $i$ th disaster area before deadline $l t_{i}$, which depends on the emergency degree in the disaster area. The deadline corresponds to a hard time window in vehicle routing problem.

2.2. The Analysis of System Risk. It is challenging work that the rescue center could respond to unexpected disasters timely. On one hand, the security risk of road conditions becomes a very important factor of whether to choose the path. If the hidden danger in a particular path is tremendous for rescuers and rescue vehicles, the possibility of this path to be selected will decrease. On the other hand, travel time of the vehicle will be delayed to some extent because the unexpected accidents on the road make driving conditions limited. So we define a new conception of efficiency risk to weigh the risk of arrival time beyond the deadline. The difference between security risk and efficiency risk lies in respective role. The security risk determines whether a path is to be selected as alternative path while efficiency risk weighs an optimal route among available paths.

The efficiency risk can be measured directly by travel time, so the utility function of disaster area can reflect it in the model. However, it is difficult to scale the security risk directly. In order to calculate the utility of security risk, the risk coefficient $R_{i j}\left(R_{i j} \in[0,1]\right)$ is introduced. Owing to bringing negative effect on whole system, higher security risk is to reduce the total utility increasingly. Similarly, with the increase of $R_{i j}$ from 0 to 1 , the security risk becomes more and more tremendous. In particular, there is no security risk in the road when $R_{i j}$ equals 0 . And the path between $i$ and $j$ is not available when $R_{i j}$ equals 1 . According to the literature [10], we define a loss function $u_{i j}^{s}$. The relationship between $u_{i j}^{s}$ and $R_{i j}$ can be formulated as follows:

$$
u_{i j}^{s}=h e^{R_{i j}}-h .
$$

And the curve graph is presented in Figure 2. From Figure 2, with the increase of risk coefficient, the amplitude of loss function value is larger and larger.

\section{The Model of EVSP}

3.1. The Fuzzy Model. With respect to the uncertain road conditions, the velocity of each relief vehicle may be fuzzy. Here, triangular fuzzy number is used to depict the fuzzy variables. For instance, the vehicle velocity in the path between $i$ and $j$ can be replaced by $v_{i j}=\left(v_{i j 1}, v_{i j 2}, v_{i j 3}\right)$, where $v_{i j 1}$ represents the minimal velocity in path $(i, j), v_{i j 2}$ is the most probable velocity, and $v_{i j 3}$ means the maximal velocity. So the travel time on road $(i, j)$ can be calculated by 


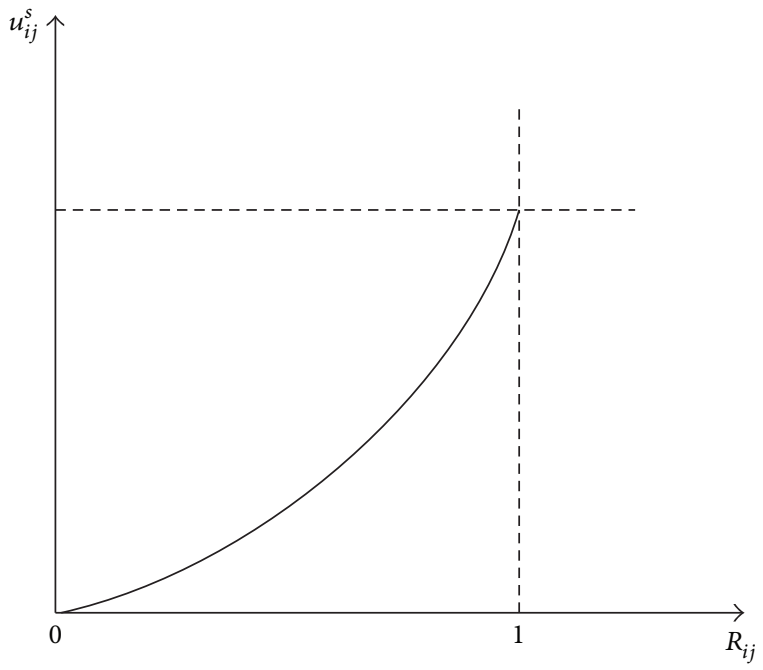

FIGURE 2: Loss function.

$\tilde{t}_{i j}=\left(d_{i j} / v_{i j 3}, d_{i j} / v_{i j 2}, d_{i j} / v_{i j 1}\right)$ against the basic arithmetic operations of triangular fuzzy number [11]. Based on the mathematical model in [6], a fuzzy model of EVSP can be formulated as follows:

$\max \quad U=\sum_{i=1}^{n} \beta_{i} u_{i}-\sum_{k=1}^{K} \sum_{j=1}^{n} \sum_{i=0}^{n} x_{i j k} u_{i j}^{s}-b \sum_{k=1}^{K} \sum_{j=1}^{n} \sum_{i=0}^{n} c_{k} r_{i} d_{i j} x_{i j k}$,

s.t. $\quad t_{j}=\sum_{i=0}^{n} x_{i j k}\left(t_{i}+\frac{d_{i j}}{\widetilde{v}_{i j}}+s_{i}\right) \quad\left(t_{0}=0, s_{0}=0, i \neq j\right)$

$\sum_{j=1}^{n} \sum_{k=1}^{K} x_{0 j k}=\sum_{i=1}^{n} \sum_{k=1}^{K} x_{i 0 k}=K$

$\sum_{j=1}^{n} \sum_{k=1}^{K} x_{i j k}=1 \quad(i=0,1, \ldots, n)$

$\sum_{i=0}^{n} \sum_{k=1}^{K} x_{i j k}=1 \quad(j=1,2, \ldots, n)$

$\sum_{j=1}^{n} x_{0 j k}=\sum_{i=1}^{n} x_{i 0 k}=1 \quad(k=1,2, \ldots, K)$

$\sum_{i=0}^{n} \sum_{j=1}^{n} x_{i j k} r_{i} \leq q_{k} \quad(k=1,2, \ldots, K)$

$0 \leq t_{i} \leq l t_{i} \quad(i=1,2, \ldots, n)$

$u_{i}=u_{0} \times \frac{u_{0}}{l t_{i}} \times e^{-t_{i}} \quad(i=1,2, \ldots, n)$

$u_{i j}^{s}=h e^{R_{i j}}-h \quad(i=1,2, \ldots, n ; j=1,2, \ldots, n)$

$$
x_{i j k}= \begin{cases}1 & \text { if vehicle } k \text { traverse from } i \text { to } j \\ 0 & \text { else. }\end{cases}
$$

Equation (2) is the objective of this model, including the total utility of all the disaster areas, loss function utility of all road security risks, and the utility value converted by transportation cost. Equation (3) is to calculate the time point when the vehicle arrives at disaster area $j$. Equation (4) means that the number of vehicles departing from the rescue center and returning back to the center is, respectively, equal to the total vehicles. Equations (5) and (6) present that each disaster area must be served by exactly one vehicle. Equation (7) means that each vehicle departing from the rescue center must return to the center. Constraint (8) restricts the capacity of each vehicle to the specified scope. Equation (9) ensures the arrival time within limited scope. Equation (10) is used to calculate the actual utility disaster areas received in emergency. Finally, (11) is loss function of security risk mentioned above.

3.2. The Crisp Model. According to the property of triangular fuzzy number [12], (3) of the above model can be represented as a fuzzy chance-constrained equation as follows:

$$
\operatorname{pos}\left\{t_{j}=\sum x_{i j k}\left(t_{i}+\frac{d_{i j}}{\widetilde{v}_{i j}}+s_{i}\right)\right\} \geq \gamma
$$

where $\gamma$ is a confidence coefficient, which is distributed in $[0,1]$. On the basis of the arithmetic operations of triangular fuzzy number, $t_{j}$ can be expressed as $\left(\sum x_{i j k}\left(t_{i}+d_{i j} / v_{i j 3}+s_{i}\right)\right.$, $\sum x_{i j k}\left(t_{i}+d_{i j} / v_{i j 2}+s_{i}\right)$, and $\left.\sum x_{i j k}\left(t_{i}+d_{i j} / v_{i j 1}+s_{i}\right)\right)$. Hence, the fuzzy chance-constrained (13) can be converted to the crisp equivalence equation correspondingly as follows [13]:

$$
\operatorname{pos}\left\{t_{j}=\sum x_{i j k}\left(t_{i}+\frac{d_{i j}}{\widetilde{v}_{i j}}+s_{i}\right)\right\}=\mu_{\sum x_{i j k}\left(t_{i}+d_{i j} / \widetilde{v}_{i j}+s_{i}\right)}\left(t_{j}\right),
$$

where $\mu_{\sum x_{i j k}\left(t_{i}+d_{i j} / \widetilde{v}_{i j}+s_{i}\right)}\left(t_{j}\right)$ is a membership function of triangular fuzzy number $\sum x_{i j k}\left(t_{i}+d_{i j} / \widetilde{v}_{i j}+s_{i}\right)$.

Lemma 1. If $\operatorname{pos}\left\{t_{j}=\sum x_{i j k}\left(t_{i}+d_{i j} / \widetilde{v}_{i j}+s_{i}\right)\right\} \geq \gamma$ holds, the following inequality must be tenable:

$$
\begin{aligned}
& t_{j} \geq \sum x_{i j k}\left[t_{i}+\frac{(1-\gamma) d_{i j}}{v_{i j 1}}+s_{i}\right]+\sum x_{i j k}\left(t_{i}+\frac{\gamma d_{i j}}{v_{i j 2}}+s_{i}\right) \\
& t_{j} \leq \sum x_{i j k}\left[t_{i}+\frac{(1-\gamma) d_{i j}}{v_{i j 3}}+s_{i}\right]+\sum x_{i j k}\left(t_{i}+\frac{\gamma d_{i j}}{v_{i j 2}}+s_{i}\right) .
\end{aligned}
$$

Proof. From (13) and (14), we can derive $\mu_{\sum x_{i j k}\left(t_{i}+d_{i j} / v_{i j}+s_{i}\right)}\left(t_{j}\right) \geq \gamma$ easily. That is, $t_{j}$ is within $\gamma$ 
level set of $\sum x_{i j k}\left(t_{i}+d_{i j} / \widetilde{v}_{i j}+s_{i}\right)$. In addition, the $\gamma$ level set of $\sum x_{i j k}\left(t_{i}+d_{i j} / \widetilde{v}_{i j}+s_{i}\right)$ can be described as

$$
\begin{gathered}
{\left[(1-\gamma) \sum x_{i j k}\left(t_{i}+\frac{d_{i j}}{v_{i j 1}}+s_{i}\right)+\gamma \sum x_{i j k}\left(t_{i}+\frac{d_{i j}}{v_{i j 2}}+s_{i}\right),\right.} \\
\left.(1-\gamma) \sum x_{i j k}\left(t_{i}+\frac{d_{i j}}{v_{i j 3}}+s_{i}\right)+\gamma \sum x_{i j k}\left(t_{i}+\frac{d_{i j}}{v_{i j 2}}+s_{i}\right)\right] .
\end{gathered}
$$

Therefore,

$$
\begin{aligned}
& t_{j} \geq \sum x_{i j k}\left[t_{i}+\frac{(1-\gamma) d_{i j}}{v_{i j 1}}+s_{i}\right]+\sum x_{i j k}\left(t_{i}+\frac{\gamma d_{i j}}{v_{i j 2}}+s_{i}\right) \\
& t_{j} \leq \sum x_{i j k}\left[t_{i}+\frac{(1-\gamma) d_{i j}}{v_{i j 3}}+s_{i}\right]+\sum x_{i j k}\left(t_{i}+\frac{\gamma d_{i j}}{v_{i j 2}}+s_{i}\right) .
\end{aligned}
$$

From Lemma 1 , one can learn a value range of $t_{j}$. For the sake of an exact expression, we define the optimistic coefficient of decision makers as $\theta$, which distributed in $[0,1]$. More optimistic the decision makers become, higher the $\theta$ value is. And the $t_{j}$ can be changed to

$$
\begin{gathered}
t_{j}=\left[\sum x_{i j k}\left(t_{i}+\frac{(1-\gamma) d_{i j}}{v_{i j 1}}+s_{i}\right)+\sum x_{i j k}\left(t_{i}+\frac{\gamma d_{i j}}{v_{i j 2}}+s_{i}\right)\right] \\
+\theta\left\{\left[\sum x_{i j k}\left(t_{i}+\frac{(1-\gamma) d_{i j}}{v_{i j 3}}+s_{i}\right)\right.\right. \\
\left.+\sum x_{i j k}\left(t_{i}+\frac{\gamma d_{i j}}{v_{i j 2}}+s_{i}\right)\right] \\
-\left[\sum x_{i j k}\left(t_{i}+\frac{(1-\gamma) d_{i j}}{v_{i j 1}}+s_{i}\right)\right. \\
\left.\left.+\sum x_{i j k}\left(t_{i}+\frac{\gamma d_{i j}}{v_{i j 2}}+s_{i}\right)\right]\right\} .
\end{gathered}
$$

After simplification, namely,

$t_{j}=\sum x_{i j k}\left[t_{i}+\gamma \frac{d_{i j}}{v_{i j 2}}+(1-\gamma) d_{i j} \frac{\theta v_{i j 1}+(1-\theta) v_{i j 3}}{v_{i j 1} v_{i j 3}}+s_{i}\right]$.

So the crisp model can be shown in (20)

s.t.

$t_{j}=\sum x_{i j k}\left[t_{i}+\gamma \frac{d_{i j}}{v_{i j 2}}+(1-\gamma) d_{i j} \frac{\theta v_{i j 1}+(1-\theta) v_{i j 3}}{v_{i j 1} v_{i j 3}}+s_{i}\right]$,

$$
\left(t_{0}=0, s_{0}=0\right)
$$

constraints $(4) \sim(11)$.

\section{Particle Swarm Optimization}

EVSP is a NP problem with multiple decision variables and multiple constraints. The evolutionary computation has achieved a lot of good results for most problems in recent years, especially particle swarm optimization (PSO). Inspired by the swarm behavior of birds flocking, Eberhart and Kennedy [14] proposed the PSO algorithm. In view of the excellent property of PSO, such as a good ability of robustness and short run time, we employ PSO to solve a case of EVSP in this paper.

In PSO, based on stochastic search in a $D$-dimensional space, a particle in the swarm represents a point in the region above, namely, a feasible solution for the problem. To achieve a balance between the global and local search, inertia weight $w$ [15] was introduced in the following equation:

$$
\begin{gathered}
v_{i d}=w * v_{i d}+c_{1} * \operatorname{rand}_{1} *\left(\text { pbest }_{i d}-x_{i d}\right) \\
+c_{2} * \operatorname{rand}_{2} *\left(\text { gbest }_{d}-x_{i d}\right) \\
x_{i d}=x_{i d}+v_{i d},
\end{gathered}
$$

where $V_{i}=\left(v_{i 1}, v_{i 2}, \ldots, v_{i D}\right)$ and $X_{i}=\left(x_{i 1}, x_{i 2}, \ldots, x_{i D}\right)$ denote the velocity and position of particle $i$, respectively. pbest $_{i}=\left(\right.$ pbest $_{i 1}$, pbest $_{i 2}, \ldots$, pbest $\left._{i D}\right)$ is the best previous position of the $i$ th particle weighted by the historically best fitness value. gbest $_{d}=\left(\right.$ gbest $_{1}$, gbest $_{2}, \ldots$, gbest $\left._{D}\right)$ is the best position of whole swarm in the search space. $c_{1}$ and $c_{2}$ are two acceleration constants indicating the extent to which each particle flies toward pbest and gbest positions, respectively. rand $_{1}$ and rand $_{2}$ are two random numbers uniformly distributed in $[0,1]$.

It is very crucial for EVSP to express the particles appropriately. And $N+K-1$ encoding method [16] is adopted to express each particle. Besides, penalty function is used to develop the fitness function so as to come up to two constraints, for example, vehicle load within the given capacity and time windows. Thus, the fitness function can be changed to

$$
\begin{aligned}
\text { fitness }= & \max U-M \max \left(Q_{k}-q_{k}, 0\right) \\
& -M \max \left(t_{i}-l t_{i}, 0\right)
\end{aligned}
$$

where $Q_{k}$ represents the actual load of relief vehicle $k$. And $q_{k}$ stands for the given capacity of vehicle $k$. Moreover, $M$ is set to an infinite number.

\section{The Numerical Experiments}

5.1. Experimental Settings. There is a scenario [17] where a disaster assistance center with 3 relief vehicles needs to distribute relief commodities to 8 disaster areas. The urgency degrees of material demands, unloading time, and time windows of disaster areas are presented in Table 1. The distance between rescue center and disaster areas is showed in Table 2 . And Table 3 gives the risk coefficient of each path.

This paper sets the contrast experiments for further comparative analysis. There is no efficiency risk or security risk 
TABLE 1: The basic information of disaster areas.

\begin{tabular}{lcccc}
\hline $\begin{array}{l}\text { Disaster } \\
\text { areas }\end{array}$ & Demands & $\begin{array}{c}\text { Unloading } \\
\text { time }\end{array}$ & $\begin{array}{c}\text { Time } \\
\text { windows }\end{array}$ & $\begin{array}{c}\text { Urgency } \\
\text { degree }\end{array}$ \\
\hline 1 & 1 & 0.2 & {$[0,6]$} & 0.1 \\
2 & 2 & 0.3 & {$[0,6]$} & 0.1 \\
3 & 4 & 0.5 & {$[0,3]$} & 0.1 \\
4 & 3 & 0.4 & {$[0,7]$} & 0.1 \\
5 & 1.5 & 0.2 & {$[0,6]$} & 0.1 \\
6 & 1 & 0.5 & {$[0,5]$} & 0.1 \\
7 & 2.5 & 0.4 & {$[0,7]$} & 0.1 \\
8 & 3 & 0.4 & {$[0,9]$} & 0.3 \\
\hline
\end{tabular}

TABLE 2: The distance between rescue center and disaster areas.

\begin{tabular}{lccccccccc}
\hline$d_{i j}$ & 0 & 1 & 2 & 3 & 4 & 5 & 6 & 7 & 8 \\
\hline 0 & 0 & 20 & 80 & 65 & 70 & 80 & 40 & 80 & 60 \\
1 & & 0 & 18 & 35 & 50 & 50 & 40 & 70 & 60 \\
2 & & & 0 & 75 & 40 & 60 & 75 & 75 & 75 \\
3 & & & & 0 & 30 & 50 & 90 & 90 & 150 \\
4 & & & & & 0 & 20 & 75 & 75 & 100 \\
5 & & & & & & 0 & 70 & 90 & 75 \\
6 & & & & & & & 0 & 70 & 100 \\
7 & & & & & & & & 0 & 100 \\
8 & & & & & & & & & 0 \\
\hline
\end{tabular}

TABlE 3: The risk coefficient of each path.

\begin{tabular}{ccccccccccr}
\hline$R_{i j}$ & 0 & 1 & 2 & 3 & I/II & III & 5 & 6 & 7 & 8 \\
\hline 0 & 0 & & & & & & & & \\
1 & 0 & 0 & & & & & & & \\
2 & 0 & 0 & 0 & & & & & & & \\
3 & 0 & 0 & 0 & 0 & & & & & & \\
4 & 0 & 0 & 0 & 0 & 0 & 0 & & & & \\
5 & 0 & 0 & 0 & 0 & 0 & 0 & 0 & & & \\
6 & 0 & 0 & 0 & 0 & 0 & 0 & 0 & 0 & & \\
7 & 0 & 0 & 0 & 0 & $\mathbf{0}$ & $\mathbf{0 . 8}$ & 0 & 0 & 0 & \\
8 & 0 & 0 & 0 & 0 & 0 & 0 & 0 & 0 & 0 & 0 \\
\hline
\end{tabular}

in experiment I. In experiment II, there are efficiency risks, while there are efficiency risk and security risk in experiment III, and the only security risk coefficient between disaster areas 4 and 7 is 0.8 . Besides, the velocity is constant with 60 units for every path in experiment $\mathrm{I}$, and the fuzzy velocity of experiments II and III is given in Table 4. Moreover, the vehicle capacity is 8 units and the unit freight $c_{k}=1$. The conversion factor between transportation costs and utility $b=$ 0.1 , the initial utility of disaster areas $u_{0}=100$, the confidence coefficient $\gamma=0.8$, and the optimistic coefficient of decision makers $\theta=0.5$.

5.2. Experimental Results. The PSO was programmed in MATLAB 7.14 and experiments were performed on an Intel Core i3 $3.30 \mathrm{GHz}$ computer. The parameters used in PSO are

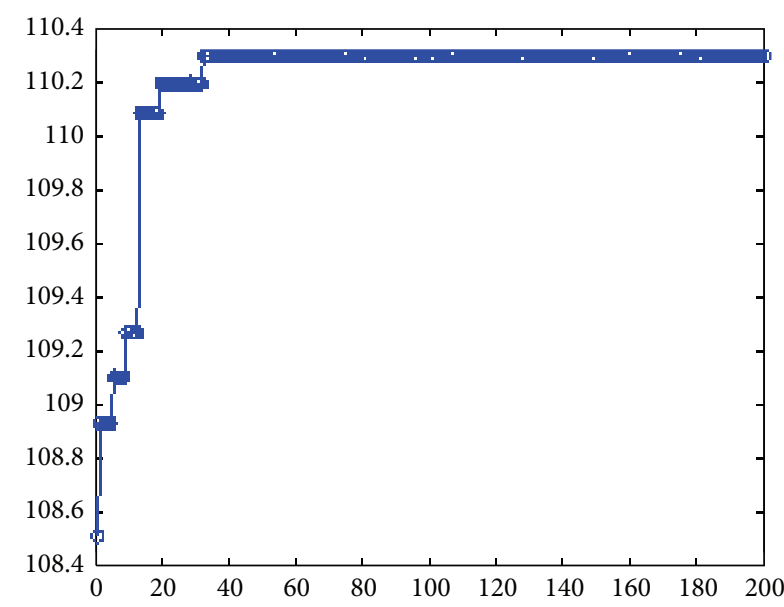

(a)

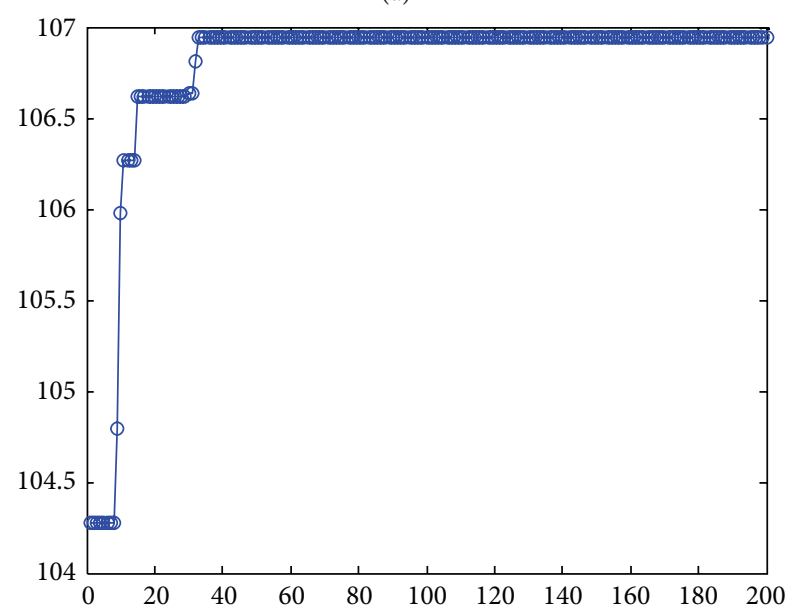

(b)

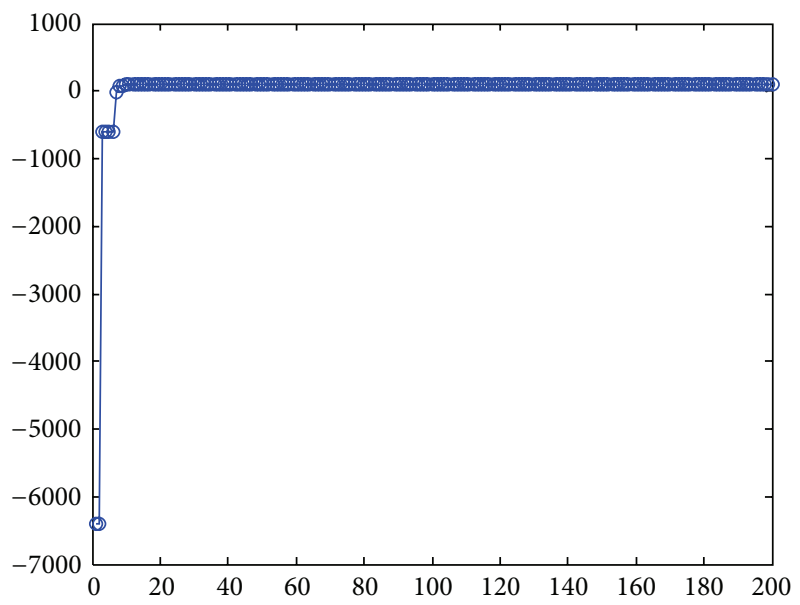

(c)

FIgURE 3: The convergence graph for experiment.

set as follows: the acceleration coefficients $c_{1}=c_{2}=2$ and the inertia weight $w=0.8$. Besides, the popular size is set to 40 and maximum iteration is set to 200 . The maximum utility discovered in experiment I is 110.2965 as Figure 3(a) shows. In experiment II, the optimal fitness value is 106.9495 and Figure 3(b) gives the convergence graph. The best result of 
TABLE 4: The fuzzy velocity.

\begin{tabular}{|c|c|c|c|c|c|c|c|c|c|}
\hline$\widetilde{v}_{i j}$ & 0 & 1 & 2 & 3 & 4 & 5 & 6 & 7 & 8 \\
\hline 0 & - & $10,10,60$ & $10,55,60$ & $10,50,60$ & $10,30,60$ & $10,58,60$ & $10,55,60$ & $10,55,60$ & $10,50,60$ \\
\hline 1 & & - & $10,40,60$ & $10,50,60$ & $10,50,60$ & $10,50,60$ & $10,50,60$ & $10,50,60$ & $10,25,60$ \\
\hline 2 & & & - & $10,50,60$ & $10,50,60$ & $10,50,60$ & $10,50,60$ & $10,50,60$ & $10,50,60$ \\
\hline 3 & & & & - & $10,55,60$ & $10,50,60$ & $10,55,60$ & $10,55,60$ & $10,55,60$ \\
\hline 4 & & & & & - & $10,55,60$ & $10,55,60$ & $10,55,60$ & $10,35,60$ \\
\hline 5 & & & & & & - & $10,55,60$ & $10,55,60$ & $10,40,60$ \\
\hline 6 & & & & & & & - & $10,55,60$ & $10,35,60$ \\
\hline 7 & & & & & & & & - & $10,35,60$ \\
\hline 8 & & & & & & & & & - \\
\hline
\end{tabular}

TABLE 5: The optimal route and utility.

\begin{tabular}{llc}
\hline Item & The optimal route & $\begin{array}{c}\text { The optimal } \\
\text { utility }\end{array}$ \\
\hline \multirow{2}{*}{ Experiment I } & $\begin{array}{l}0 \rightarrow 6 \rightarrow 5 \rightarrow 4 \rightarrow 2 \rightarrow 0 \\
0 \rightarrow 1 \rightarrow 3 \rightarrow 7 \rightarrow 0\end{array}$ & 110.2965 \\
& $0 \rightarrow 8 \rightarrow 0$ & \\
\hline Experiment & $0 \rightarrow 3 \rightarrow 2 \rightarrow 1 \rightarrow 0$ & 106.9495 \\
II & $0 \rightarrow 6 \rightarrow 5 \rightarrow 4 \rightarrow 7 \rightarrow 0$ & \\
\hline Experiment & $0 \rightarrow 8 \rightarrow 0$ & 106.6194 \\
III & $0 \rightarrow 3 \rightarrow 1 \rightarrow 7 \rightarrow 0$ & \\
\hline
\end{tabular}

experiment III is 106.6194, which is presented in Figure 3(c). The optimal vehicle route for each experiment and the corresponding time point are showed in Tables 5 and 6, respectively.

By making a comparison between experiments I and II, we can conclude the following.

(1) The optimistic coefficient of decision makers is affected by the road conditions $(\theta=0.5<1.0)$, which leads to the longer waiting time of disaster areas remarkably as a result of fuzzy velocity.

(2) The optimal vehicle route depends on road conditions. In detail, the most probable velocity between the rescue center and disaster area 1 is lowest in experiment II $\left(v_{012}=10\right)$ because of many road blocks incurred by the disaster. Considering the efficiency risk and maximum utility, the disaster assistance center has to eliminate path $(0,1)$ from the optimal route.

(3) The efficiency risk plays a negative role on the system utility. Evidently, the optimal utility discovered is reduced after considering the road conditions $(106.9495<110.2965)$. Owing to the fuzzy travel time, there exists the efficiency risk that prolongs travel time. So the total utility of emergency rescue system is reduced to some extent.

In summary, the road conditions could influence vehicle velocity directly so that there is the efficiency risk determining whether the path is chosen as one of the optimal routes.
In other words, the existence of efficiency risk brings negative effect on the entire system.

Similarly, by making a comparison between experiments II and III, we can conclude the following.

(1) The security risk determines whether a path is available in the distribution. There is a high risk coefficient between disaster areas 4 and $7\left(R_{47}=0.8\right)$. The rescue center must attend to the safety of rescue workers and abandon path $(4,7)$ eventually.

(2) By considering the utility of each part, the emergency rescue system becomes flexible when compared with the traditional one only optimizing the total cost or distance. For example, the optimal route scheme is reenacted after discarding path $(4,7)$ because of its high security risk. We can calculate the travelled distance of experiments II (583 units) and III (620 units) readily. It is apparent that the transportation cost soars due to bypassing the optimal path $(4,7)$. However, the travel time is shortened significantly in experiment III, which compensates for the utility of entire system.

(3) The security risk also results in negative effect on the entire system. Considering the security risk in the distribution, the total utility is lower because the security risk restricts a path to be selected as one of the available routes. The incremental transportation cost on account of this limitation would give rise to the decrease of total utility.

In conclusion, the efficiency risk and security risk can influence the optimal route scheme substantially and bring negative effect on the emergency rescue system. It should be noted, in real world, that there exist efficiency risk and security risk usually in terms of the road conditions in emergency, especially in natural disaster areas (e.g., aftershock and landslide).

\section{Conclusions}

The flexible emergency rescue system is presented in this paper, including disaster assistance center, relief vehicles, and disaster areas. The utility of each part is calculated in the objective function. Based on the previous study works, 
TABLE 6: The time point.

\begin{tabular}{lcccccccc}
\hline Disaster area & 1 & 2 & 3 & 4 & 5 & 6 & 7 & \\
\hline Time point & & & & & & & & \\
I & 0.33 & 3.93 & 1.12 & 2.87 & 2.33 & 0.67 & 3.12 & 1.00 \\
II & 5.24 & 4.37 & 1.80 & 4.12 & 3.38 & 1.05 & 6.47 \\
III & 3.27 & 5.61 & 1.80 & 4.11 & 3.38 & 1.05 & 5.40 \\
\hline
\end{tabular}

the uncertain road conditions are involved in the extended model. Then, two new conceptions, efficiency risk and security risk, are introduced to weigh the influence of road conditions on the system utility. Besides, we adopt triangular fuzzy number to denote the fuzzy vehicle velocity and travel time. And a fuzzy model is built by fuzzy variables and converted to the crisp model on the grounds of the property of triangular fuzzy number. In order to address this problem, PSO algorithm is used to maximize the total utility in the numerical experiments. Significantly, this proposed mathematical model is validated by the experimental results.

It should be noted that we can use utility to evaluate an emergency rescue system appropriately by integrating the transportation cost with travel time. Nevertheless, it is interesting to take more than one goal into account.

\section{Conflict of Interests}

The authors declare that there is no conflict of interests regarding the publication of this paper.

\section{Acknowledgments}

This work is supported by the National Natural Science Foundation of China (Grant nos. 71271140, 71001072, 71240015, and 71471158), the Natural Science Foundation of Guangdong Province (Grant nos. S2012010008668 and 9451806001002294 ), Social Science Research Program in Universities in Guangdong Province (Grant no. 10WYXM051), the Science and Technology Planning Project of Guangdong Province (Grant no. 2012B040305010), and the Shenzhen University Teaching Reform Project (Grant nos. JG2014003 and JG2012010).

\section{References}

[1] L. Özdamar, E. Ekinci, and B. Küçükyazici, "Emergency logistics planning in natural disasters," Annals of Operations Research, vol. 129, no. 1-4, pp. 217-245, 2004.

[2] J. Tian, W.-Z. Ma, Y.-L. Wang, and K.-L. Wang, "Emergency supplies distributing and vehicle routes programming based on particle swarm optimization," System Engineering Theory and Practice, vol. 31, no. 5, pp. 898-906, 2011.

[3] J. B. Sheu, "An emergency logistics distribution approach for quick response to urgent relief demand in disasters," Transportation Research Part E: Logistics and Transportation Review, vol. 43 , no. 6 , pp. 687-709, 2007.

[4] H. L. Sun, Z. J. Zhou, and Y. F. Xue, "Emergency locationrouting problem with uncertain demand under path risk," Journal of Shanghai Jiaotong University, vol. 47, no. 6, pp. 962966, 2013.
[5] G. Ji and C. Zhu, "A study on emergency supply chain and risk based on urgent relief service in disasters," Systems Engineering Procedia, vol. 5, pp. 313-325, 2012.

[6] X. B. Gan, Y. Wang, Y. Yu, and B. Niu, "An emergency vehicle scheduling problem with time utility based on particle swarm optimization," in Intelligent Computing Theories and Technology, pp. 614-623, Springer, Berlin, Germany, 2013.

[7] T. Yamada, "A network flow approach to a city emergency evacuation planning," International Journal of Systems Science, vol. 27, no. 10, pp. 931-936, 1996.

[8] A. Tuson, R. Wheeler, and P. Ross, "An evolutionary/metaheuristic approach to emergency resource redistribution in the Developing World," in Artificial Neural Nets and Genetic Algorithms, pp. 329-332, Springer, Vienna, Austria, 1998.

[9] W. Yi and A. Kumar, "Ant colony optimization for disaster relief operations," Transportation Research Part E: Logistics and Transportation Review, vol. 43, no. 6, pp. 660-672, 2007.

[10] T. P. Hsu and G. H. Lu, "Development of the minimal risk routing model for post earthquake traffic management," Bulletin of the College of Engineering, National Taiwan University, vol. 85, pp. 33-48, 2002.

[11] Z.-Z. Jiang and D.-W. Wang, "Fuzzy programming model and algorithm of logistics distribution vehicle routing problem," Journal of System Simulation, vol. 18, no. 11, pp. 3301-3304, 2006.

[12] S. C. Fang and D. W. Wang, Fuzzy Mathematics and Fuzzy Optimization, Science Press, Beijing, China, 1997.

[13] X. Y. Zhao and D. W. Wang, "Fuzzy chance constrained programming model for bi-level distribution network design in the supply chain," Control Theory \& Applications, vol. 19, no. 2, pp. 249-252, 2002.

[14] R. Eberhart and J. Kennedy, "A new optimizer using particle swarm theory," in Proceedings of the 6th International Symposium on Micro Machine and Human Science, pp. 39-43, October 1995.

[15] Y. Shi and R. Eberhart, "A modified particle swarm optimizer," in Proceedings of the IEEE International Conference on Evolutionary Computation (ICEC '98), pp. 69-73, May 1998.

[16] X. B. Gan, Y. Wang, S. H. Li, and B. Niu, "Vehicle routing problem with time windows and simultaneous delivery and pick-up service based on MCPSO," Mathematical Problems in Engineering, vol. 2012, Article ID 104279, 11 pages, 2012.

[17] G. R. Zhang and Y. Zhang, "Emergency logistics distribution VRP based on partical swarm optimization," Value Engineering, vol. 34, pp. 9-10, 2011. 


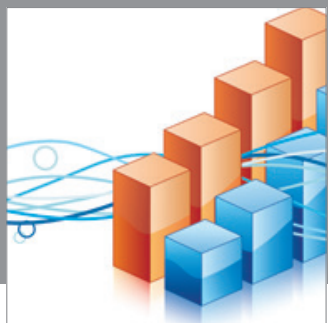

Advances in

Operations Research

mansans

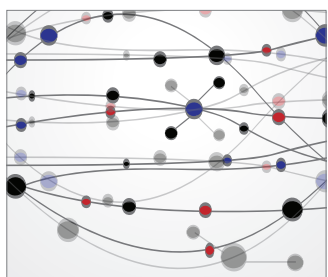

The Scientific World Journal
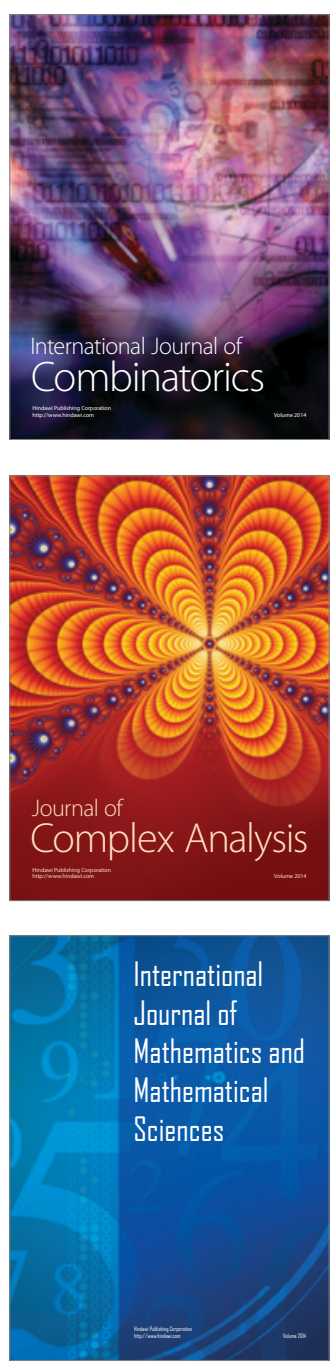
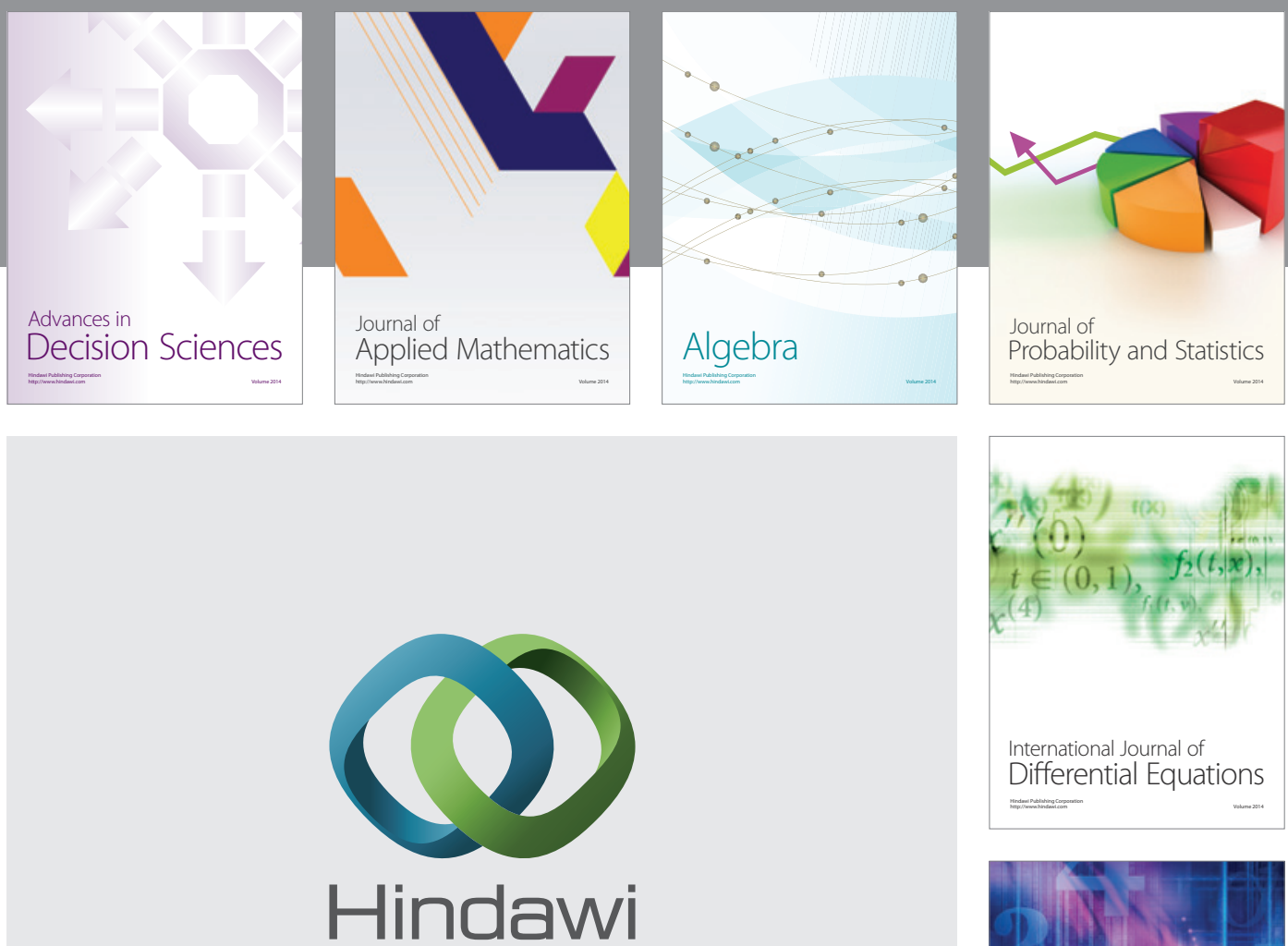

Submit your manuscripts at http://www.hindawi.com
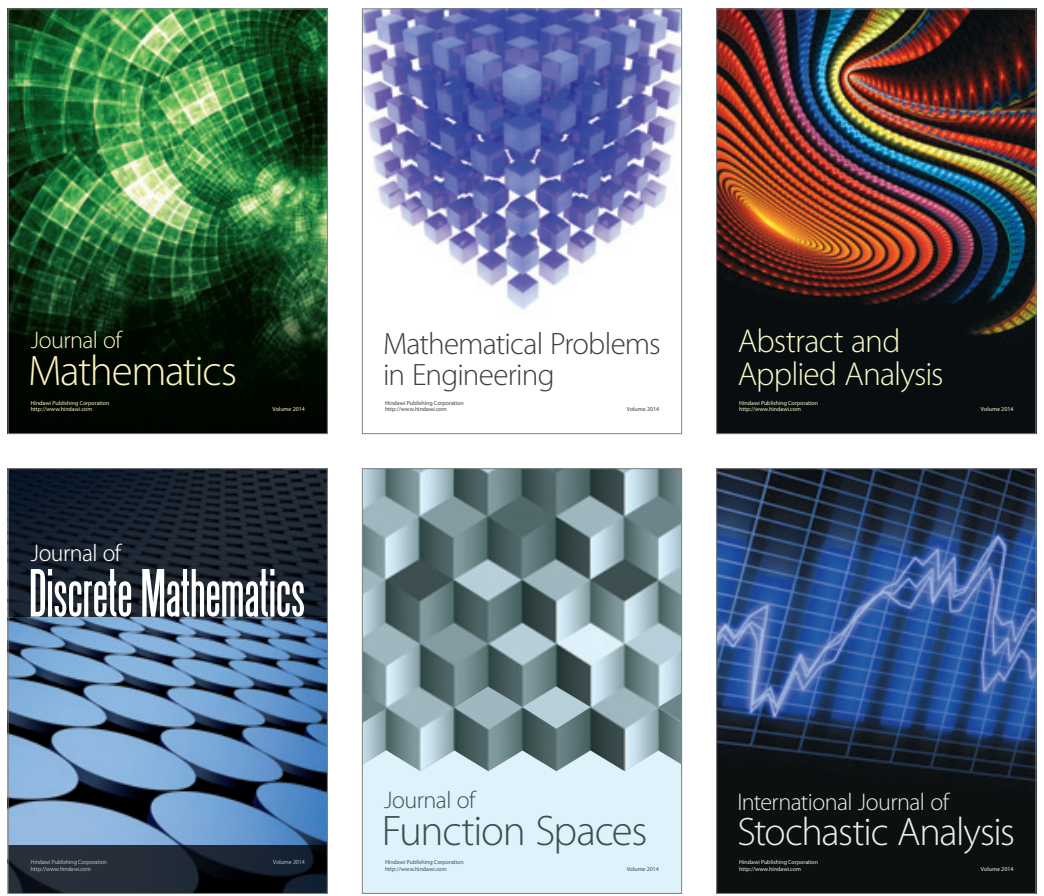

Journal of

Function Spaces

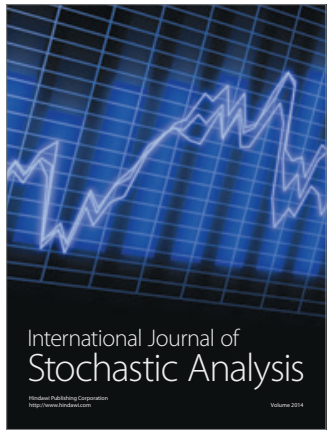

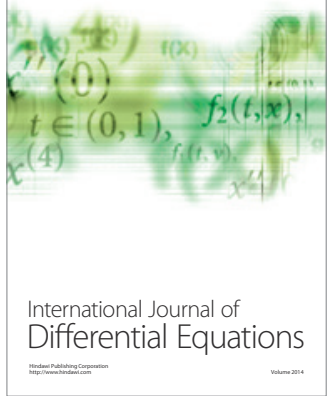
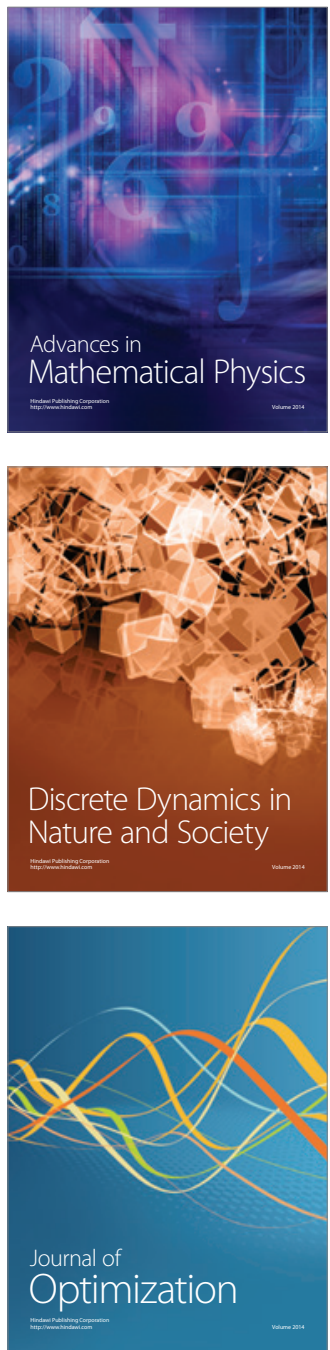